\title{
Research on the Secondary Development of Revit Software
}

\author{
Chen Nan* \\ Information and Control Engineering Academy, \\ Shenyang Jianzhu University \\ Shen Yang, China \\ e-mail: arnold0110@sina.com \\ * Corresponding Author \\ Kan Fenglong \\ Information and Control Engineering Academy, \\ Shenyang Jianzhu University \\ Shen Yang, China \\ Wang Xin \\ Information and Control Engineering Academy, \\ Shenyang Jianzhu University \\ Shen Yang, China \\ Wang Changtao \\ Information and Control Engineering Academy, \\ Shenyang Jianzhu University \\ Shen Yang, China
}

\begin{abstract}
BIM (Building Information Modeling) technology is based on "information" as the core, Throughout the building's full life cycle, Its emergence has set off a new revolution in the construction industry. And the informationization development of building industry to a large extent depends on the degree of informatization construction phase. Objective: Study of Revit software secondary development process, realize simple function plug-ins. Methods: Using the Visual Studio tools with c \# programming language to Revit software for secondary development. Results : Create a simple external command and executes commands in Revit. Conclusion : On the basis of summarizing the existing BIM technology and virtual construction technology, starting from the construction present situation and the actual demand, Analysis and research on the application of BIM in construction engineering, the combination of Revit software, secondary development to realize the BIM model for building the information access of graphic data and parameters in the model and implementation step of continuous command simplified one key function of the operation.
\end{abstract}

Keywords-Building Information Modeling(BIM);Revit; the secondary development; Visual Studio;research;

\section{INTRODUCTION}

With the rapid development of science and technology and the acceleration of the process of information, Our country has become one of the largest construction market in the world, However, it is not matched to the construction industry, the technology of the field of technology update is very slow. There are many problems in the traditional construction industry, Such as the loss of

\author{
Qi Ning \\ The School Library \\ Shenyang Jianzhu University \\ Shenyang China \\ Liu Xiyang \\ Information and Control Engineering Academy, \\ Shenyang Jianzhu University \\ Shen Yang, China \\ Mao Yongming \\ Information and Control Engineering Academy, \\ Shenyang Jianzhu University \\ Shen Yang, China \\ Wang Bin \\ Northeastern University at Qinhuangdao \\ Qinhuangdao, China
}

information, the expression of fuzzy, low efficiency and serious loss of material, For this urgent need a new kind of technology and ideas to change this situation. Based on this background, BIM (Building Information Modeling) technology arises at the historic moment, Among them Autodesk Revit series of software in which the most widely used, And it has strong ability of second development.

\section{REVIT SOFTWARE PROFILE}

At this stage in the international academic community and software development in the field of BIM technology has been unanimously affirmed. BIM technology can save a lot of time and money and reduce the rework in the engineering construction industry, Increase productivity. Of course, as a technology, it is necessary to support the implementation of the application software to support it. And the current more mature based on CAAD development of the BIM tool software [1]: Graphisoft company in 1982 began research and development of ArchiCAD, Bentley Microstation, TriFoma and Revit Autodesk, launched in 1986, Both of them have been certified by IFC2x 2 standards, Information sharing and integrated management of the entire building life cycle can be supported. Revit software is based on the theory of BLM-BIM to guide the development of engineering software, to achieve the sharing of different professional information and related [2]. It was acquired by Autodesk in 2002, Its predecessor was founded in 1997 by Pro/E Software Engineer Technology Revit company. Pro/E company is a leader in the field of mechanical industry $3 \mathrm{D}$ 
CAD, Inspired by Revit, the company has invented a new type of parametric engine for the construction industry, And study the model based modeling method. Revit software uses parametric modeling technology, although the internal data structure used by the two dimensional design software such as AutoCAD is different, but the two can carry out data exchange. Since Revit was acquired by Autodesk company, it has become the main research product line, the current development version has been upgraded to Revit 2016.

Autodesk's Revit has three series: Revit Architecture, Revit Structure and Revit MEP, Respectively correspond to the building, structure and water heating professional field, Almost all aspects of the life cycle of buildings. Although the various software tools division of labor, But its working mechanism is roughly the same, The core of the building is parametric architectural primitives. By the parameters provided by the parameters of the engine modification technology to achieve the associated changes can be achieved, "a change, everywhere update" effect.

Compared with the traditional CAD platform for 3D modeling and operation, the Revit software based on the concept of BIM has a powerful and sophisticated 3D modeling technology. Revit API(Application Prograrmming Interface) is powerful. Applies to the secondary development.

Atuodesk Revit software has the following characteristics:

(1) Powerful visual modeling features: the building, structure and water heating and other professional design is very good combination, the formation of a consistent three-dimensional visualization building model, and has a variety of user-friendly data conversion interface, the design of traditional professional design software to import the software, to achieve information sharing and collaborative work.

(2) 2D-3D easy conversion: the process of building design is not only to create a three-dimensional model of the process, but also to draw the process of flat vertical profile drawings and three-dimensional expression. The software the 3D model building and its horizontal and vertical section drawings tied, the program design and graphics performance of the combined, so that designers can switch freely in 3D model and 2D drawings. And for the more complex structures, it can obtain the profile of arbitrary position and analyze the design area.

(3) The design has gradually deepened: Revit use 3D visualization technology and data management, can truly reflect the various physical properties of the building components, in the early stage of the program designers can temporarily ignore these attributes, but with the design depth of the construction project, and then gradually add or repair the corresponding component properties, until meet the requirements of construction drawings.

(4) The update function of the view: the parameters modification technology provided by the Revit parameters, and the related changes in the model, when the model is modified, it will cause the real time modification of all view drawings and material list.

(5) Architectural primitives can be reused: Revit comes with a wealth of architectural elements in the form of architectural design elements; in addition, Revit also allows users to customize the building components, that is, according to the designer need to design the corresponding components or build their own family, in the architectural design of Chinese sources.

\section{THE SECONDARY DEVELOPMENT OF REVIT SOFTWARE}

Revit software using the interface is very friendly. With experience in AutoCAD software, it can quickly get started, if you have a certain programming based and API use method, you can according to the need to develop the Revit software for the two time, to expand its function, of course, can also be the original work of the software to optimize encoding, which is the important reason we choose to use Revit 3D modeling.

Reviy API provides a variety of functions of the Revit access interface, the analysis and visualization of the application and Revit building information model of the integrated function, the user can according to their own requirements for the functional expansion of Revit. API.Net is API Revit, and all languages that are compatible with Framework2.0 Microsoft.Net can be called API, such as: VB.Net or Visual C\# Microsoft. Revit Revit SDK (Development Kit), RevitLookup (Sofeware SDK), API Revit use of the Revit, the development of reference documents, the program loading tools and a large number of sample code, the basic cover of the API usage, so that developers can access to the building model of the components, to the extent of the software users, to a large extent, improve work efficiency.

Usually the use of Revit software for architectural design is achieved through the menu or toolbar commands, while Revit also provides application programming interface API, so that the external program through API can manipulate and access Revit. API.NET is API Revit, so as long as the.NET Framework Microsoft 2 compatible language can be fully invoked API, such as VB.NET, Visual C\# Microsoft language. API Revit provides access to the various functions of Revit, the integration of analysis and visualization applications and building information model, users can expand the Revit corresponding functions according to their own needs, and as a software developer can also be achieved at any time to access all information models, query, change component properties and create new components.

In the secondary development of Revit software, needs to meet three conditions: Revit products, Revit SDK and development tools Visual Studio. The Revit products of Autodesk company include: Autodesk Revit Architecture used in architectural design, Autodesk Revit Structure and Autodesk Revit MEP (Mechanical, Electrical, Plumbing).for the structural model. This project mainly uses the first production.

This paper mainly uses C\# language to develop the tool, and uses it in the Visual Studio platform to expand the function of Revit software. There are two main ways to develop its development: one is the external command (Command External), to add a Revit command, the user clicks the button to start the implementation; the other is the external application (Application External) mode, for the addition of menu and tool bar or other beginning of the command, the Revit can run automatically when starting or closing. The process of its development is shown in figure 1 . 
For the development of external commands, you need to create a new class, and derived from the IExternalCommand interface, and then overloaded IExternalCommand.Execute () method, add user code to achieve command function. Execute () method has three parameters, ExternalCommandData, string, ElementSet, ref, respectively, representing the application process and the document object to obtain the parameters, and the parameters of the outgoing parameters, and the object set. The creation process of the external application is similar to the external command, you can add a Ribbon button or control, but the new class is derived from the IExternalApplication interface, you need to implement the OnStartup () and OnShutdown () method, the command is executed when the need to shut down and restart Revit.exe.

In this paper, the latest 2015 version of Revit SDK (Revit SDK is the Revit application software development kit). Which include: beginners to explain the document,

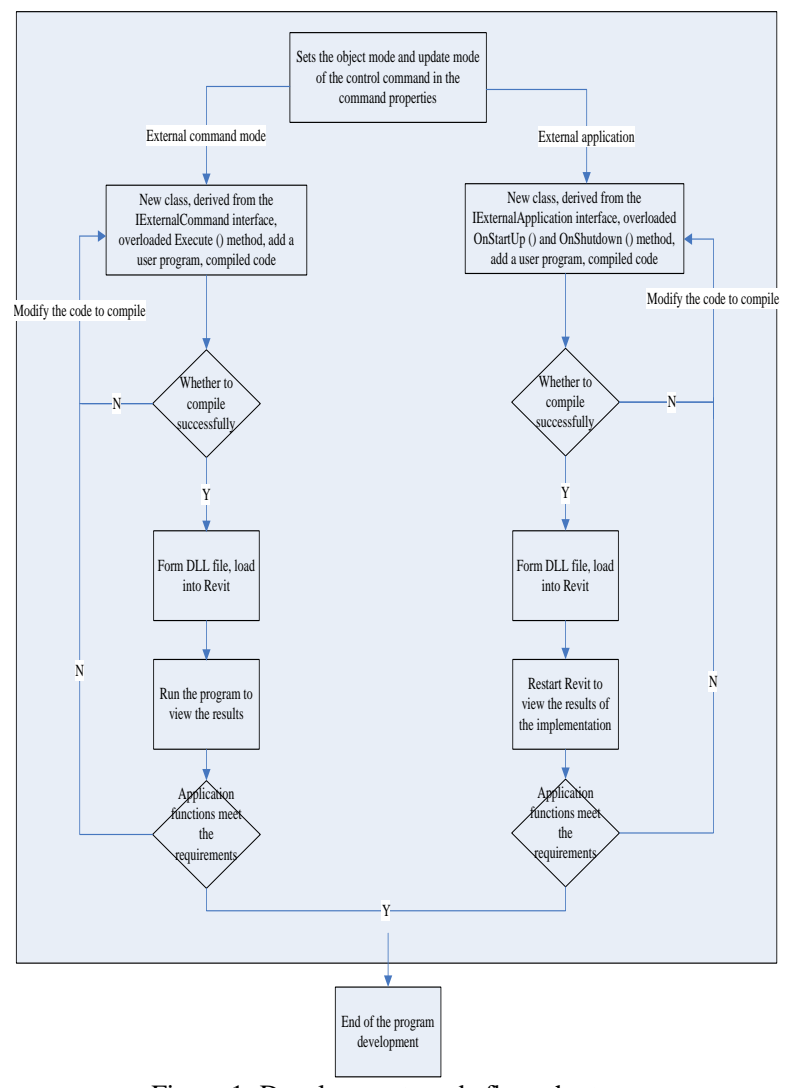

Figure 1. Development mode flow chart

developers often help reference documents, examples of program code and other information. At the same time in the SDK 2015 Revit also provides the two time to develop practical Gadget: one is Manager Add-in, the function is to load the user development of the Revit into the RevitLookup tool set, you can help users quickly learn API use, check the contents of the elements of the Revit model RevitAddInUtility.chm e-books, mainly Revit software installation information and modify.Addin file.

On the basis of mastering Revit software, combining with the API Revit interface, Revit SDK supports custom function development. Users by reading the relevant help documentation and source program sample, you can find the details of the 2015SDK interface in the previous version of the API. Specific system development process as shown in figure 2 .

\section{CONCLUSIONS}

In this paper, the Revit software secondary development process has carried on the simple discussion, for the future more in-depth study of Revit software secondary development laid a solid foundation. By developing a Revit secondary plug-in to improve the efficiency of its function, is one of effective ways to promote the development of BIM. How with secondary development technologies to make BIM technology efficient technology means, to the majority of researchers study and discussion.
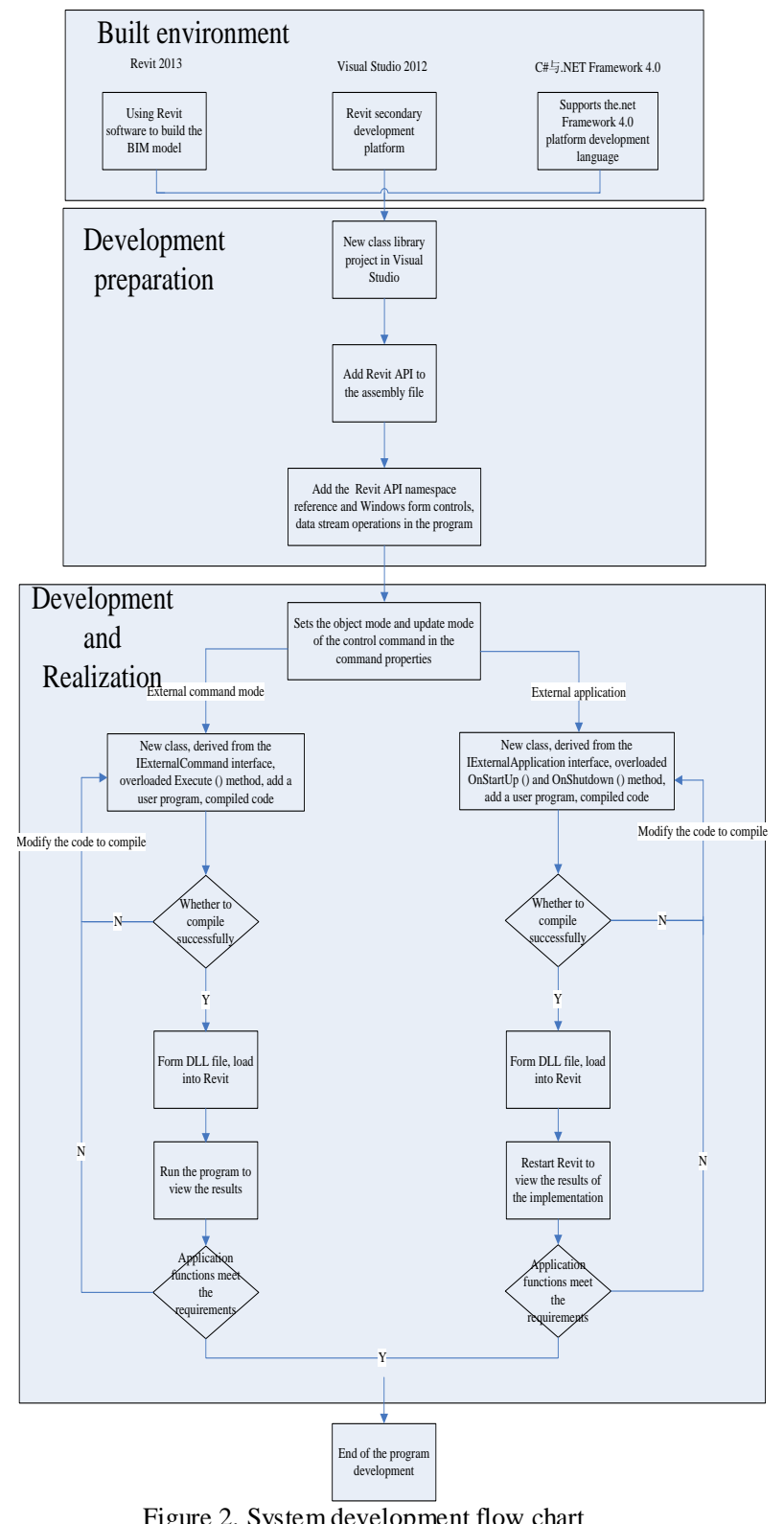

\section{REFERENCES}

[1] Jeff Stephen. Modeling For Good Performance Building Information Modeling Facilitates Greener Building [M]. Eco building pulse, 2008,48 (2),1-22. 
[2] Hartmann, T., J. Gao, and M. Fischer, Areas of application for 3D and 4D models on construction projects[J]. Journal of construction Engineering and Management, 2008. 134(10): pp.776-785.

[3] Myoung Bae Seo, Ki Beom Ju. A Study on the Interoperability between 2D Drawings and BIM-Based 3D Drawings. Scientific Research Publishing,2013,1(5)pp.10-14.

[4] Xiaobiao Lv, Yanyan Huang, Yiquan Zou. Study on BIM Technology Teaching Under The Background of Threedimensional Design. 2nd International Conference on Science and Social Research,2013,pp.50-53.

[5] Wang Xin,Guo Lili,Ma Lina. Cooperative Spectrum Sensing Algorithm Based on Second User Selection and Random Forest Classification[J].ICIC Express Letters, Part B: Applications, 2015, 6(3):845-850.

[6] Ehsan Shourangiz, Mohamad Ibrahim Mohamad, Mahmoud Shakouri Hassanabadi, Seyed Saee d Banihashemi, Mohsen
Bakhtiari, Mohsen Torabi. Flexibility of BIM towards Design Chang e. International Conference on Construction and Project Management, Singapore,2011,pp.79-83.

[7] David J. Harrington. The Implementation of BIM Standards at the Firm Level[J].Structures Congress, 2010,pp.1645-1651.

[8] Thomas W. Hartmann, P.E.BIM-BAM-BOOM! More Bang for your BIM buck[J].Structures Congress, 2010,pp. 1672-1675.

[9] Wang Xin, Huang Kuan, Gao Zhijun. The Primary Users' Signals Recognition Algorithm in Cognitive Radio Networks via KPCA and Random Forest[J].ICIC Express Letters, 2015, 9(4):1083-1088.

[10] Na Lu, Thomas Korman. Implementation of Building Information Modeling(BIM) in Modular Construction: Benefits and Challenges[J].Construction Research Congress, 2010,pp.11361145 . 